\title{
Revisiting the Westerlund 2 field with the HESS telescope array
}

HESS Collaboration, A. Abramowski ${ }^{4}$, F. Acero ${ }^{15}$, F. Aharonian ${ }^{1,13,35}$, A. G. Akhperjanian ${ }^{2,35}$, G. Anton ${ }^{16}$, A. Barnacka ${ }^{24,7}$, U. Barres de Almeida ${ }^{8, \star}$, A. R. Bazer-Bachi ${ }^{3}$, Y. Becherini ${ }^{12}$, J. Becker $^{21}$, B. Behera ${ }^{14}$, K. Bernlöhr ${ }^{1,5}$, A. Bochow ${ }^{1}$, C. Boisson ${ }^{6}$, J. Bolmont ${ }^{19}$, P. Bordas ${ }^{18}$, V. Borrel ${ }^{3}$, J. Brucker ${ }^{16}$, F. Brun ${ }^{19}$, P. Brun ${ }^{7}$, T. Bulik ${ }^{29}$, I. Büsching 9 , T. Boutelier ${ }^{17}$, S. Casanova ${ }^{1}$, M. Cerruti ${ }^{6}$, P. M. Chadwick ${ }^{8}$, A. Charbonnier ${ }^{19}$, R. C. G. Chaves ${ }^{1}$, A. Cheesebrough ${ }^{8}$, J. Conrad ${ }^{31}$, L.-M. Chounet ${ }^{10}$, A. C. Clapson ${ }^{1}$, G. Coignet ${ }^{11}$, M. Dalton ${ }^{5}$, M. K. Daniel ${ }^{8}$, I. D. Davids ${ }^{22,9}$, B. Degrange ${ }^{10}$, C. Deil ${ }^{1}$, H. J. Dickinson ${ }^{8}$, A. Djannati-Ataï ${ }^{12}$,

W. Domainko ${ }^{1}$, L. O'C. Drury ${ }^{13}$, F. Dubois ${ }^{11}$, G. Dubus ${ }^{17}$, J. Dyks ${ }^{24}$, M. Dyrda ${ }^{28}$, K. Egberts ${ }^{1,30}$, P. Eger $^{16}$, P. Espigat ${ }^{12}$, L. Fallon ${ }^{13}$, C. Farnier ${ }^{15}$, S. Fegan ${ }^{10}$, F. Feinstein ${ }^{15}$, M. V. Fernandes ${ }^{4}$, A. Fiasson ${ }^{11}$, A. Förster ${ }^{1}$, G. Fontaine $^{10}$, M. Füßling ${ }^{5}$, S. Gabici ${ }^{13}$, Y. A. Gallant ${ }^{15}$, L. Gérard ${ }^{12}$, D. Gerbig ${ }^{21}$, B. Giebels ${ }^{10}$, J. F. Glicenstein ${ }^{7}$, B. Glück ${ }^{16}$, P. Goret ${ }^{7}$, D. Göring ${ }^{16}$, J. D. Hague ${ }^{1}$, D. Hampf ${ }^{4}$, M. Hauser ${ }^{14}$, S. Heinz ${ }^{16}$, G. Heinzelmann ${ }^{4}$, G. Henri ${ }^{17}$, G. Hermann ${ }^{1}$, J. A. Hinton ${ }^{33}$, A. Hoffmann ${ }^{18}$, W. Hofmann ${ }^{1}$, P. Hofverberg ${ }^{1}$, M. Holleran ${ }^{9}$, S. Hoppe ${ }^{1}$, D. Horns ${ }^{4}$, A. Jacholkowska ${ }^{19}$, O. C. de Jager ${ }^{9}$, C. Jahn ${ }^{16}$, I. Jung ${ }^{16}$, K. Katarzyński ${ }^{27}$, U. Katz ${ }^{16}$, S. Kaufmann ${ }^{14}$, M. Kerschhaggl ${ }^{5}$, D. Khangulyan ${ }^{1}$, B. Khélifi ${ }^{10}$, D. Keogh ${ }^{8}$, D. Klochkov ${ }^{18}$, W. Kluźniak ${ }^{24}$, T. Kneiske ${ }^{4}$, Nu. Komin ${ }^{7}$, K. Kosack ${ }^{7}$, R. Kossakowski ${ }^{11}$, G. Lamanna ${ }^{11}$, J.-P. Lenain ${ }^{6}$, D. Lennarz ${ }^{1}$, T. Lohse ${ }^{5}$, C.-C. Lu ${ }^{1}$, V. Marandon ${ }^{12}$, A. Marcowith ${ }^{15}$, J. Masbou ${ }^{11}$, D. Maurin ${ }^{19}$, T. J. L. McComb ${ }^{8}$ M. C. Medina ${ }^{7}$, J. Méhault ${ }^{15}$, R. Moderski ${ }^{24}$, E. Moulin ${ }^{7}$, M. Naumann-Godo ${ }^{7}$, M. de Naurois ${ }^{10}$, D. Nedbal ${ }^{20}$, D. Nekrassov ${ }^{1}$, N. Nguyen ${ }^{4}$, B. Nicholas ${ }^{26}$, J. Niemiec ${ }^{28}$, S. J. Nolan ${ }^{8}$, S. Ohm ${ }^{1}$, J.-F. Olive ${ }^{3}$, E. de Oña Wilhelmi ${ }^{1}$, B. Opitz ${ }^{4}$, K. J. Orford ${ }^{8}$, M. Ostrowski ${ }^{23}$, M. Panter ${ }^{1}$, M. Paz Arribas ${ }^{5}$, G. Pedaletti ${ }^{14}$, G. Pelletier ${ }^{17}$, P.-O. Petrucci ${ }^{17}$, S. Pita ${ }^{12}$, G. Pühlhofer ${ }^{18}$, M. Punch ${ }^{12}$, A. Quirrenbach ${ }^{14}$, M. Raue ${ }^{1,34}$, S. M. Rayner ${ }^{8}$, A. Reimer ${ }^{30}$, O. Reimer $^{30}$, M. Renaud ${ }^{15}$, R. de los Reyes ${ }^{1}$, F. Rieger ${ }^{1,34}$, J. Ripken ${ }^{31}$, L. Rob ${ }^{20}$, S. Rosier-Lees ${ }^{11}$, G. Rowell ${ }^{26}$, B. Rudak ${ }^{24}$, C. B. Rulten ${ }^{8}$, J. Ruppel ${ }^{21}$, F. Ryde ${ }^{32}$,

V. Sahakian ${ }^{2,35}$, A. Santangelo ${ }^{18}$, R. Schlickeiser ${ }^{21}$, F. M. Schöck ${ }^{16}$, A. Schönwald ${ }^{5}$, U. Schwanke ${ }^{5}$, S. Schwarzburg ${ }^{18}$, S. Schwemmer ${ }^{14}$, A. Shalchi ${ }^{21}$, I. Sushch ${ }^{5}$, M. Sikora ${ }^{24}$, J. L. Skilton ${ }^{25}$, H. Sol ${ }^{6}$, G. Spengler ${ }^{5}$, Ł. Stawarz ${ }^{23}$, R. Steenkamp ${ }^{22}$, C. Stegmann ${ }^{16}$, F. Stinzing ${ }^{16}$, A. Szostek ${ }^{23,17}$, P. H. Tam ${ }^{14}$, J.-P. Tavernet ${ }^{19}$, R. Terrier ${ }^{12}$, O. Tibolla ${ }^{1}$, M. Tluczykont ${ }^{4}$, K. Valerius ${ }^{16}$, C. van Eldik ${ }^{1}$, G. Vasileiadis ${ }^{15}$, C. Venter ${ }^{9}$, J. P. Vialle ${ }^{11}$, A. Viana ${ }^{7}$, P. Vincent ${ }^{19}$, M. Vivier ${ }^{7}$, H. J. Völk ${ }^{1}$, F. Volpe ${ }^{1}$, S. Vorobiov ${ }^{15}$, S. J. Wagner ${ }^{14}$, M. Ward ${ }^{8}$, A. A. Zdziarski ${ }^{24}$, A. Zech ${ }^{6}$, H.-S. Zechlin ${ }^{4}$, Y. Fukui ${ }^{36}$, N. Furukawa ${ }^{36}$, A. Ohama ${ }^{36}$, H. Sano ${ }^{36}$, J. Dawson ${ }^{36}$, and A. Kawamura ${ }^{36}$

(Affiliations can be found after the references)

Received 28 June 2010 / Accepted 9 September 2010

\section{ABSTRACT}

Aims. Previous observations with the HESS telescope array revealed the existence of extended very-high-energy (VHE; $E>100 \mathrm{GeV}) \gamma$-ray emission, HESS J1023-575, coincident with the young stellar cluster Westerlund 2. At the time of discovery, the origin of the observed emission was not unambiguously identified, and follow-up observations have been performed to further investigate the nature of this $\gamma$-ray source. Methods. The Carina region towards the open cluster Westerlund 2 has been re-observed, increasing the total exposure to 45.9 h. The combined dataset includes $33 \mathrm{~h}$ of new data and now permits a search for energy-dependent morphology and detailed spectroscopy.

Results. A new, hard spectrum VHE $\gamma$-ray source, HESS J1026-582, was discovered with a statistical significance of $7 \sigma$. It is positionally coincident with the Fermi LAT pulsar PSR J1028-5819. The positional coincidence and radio/ $\gamma$-ray characteristics of the LAT pulsar favors a scenario where the TeV emission originates from a pulsar wind nebula. The nature of HESS J1023-575 is discussed in light of the deep HESS observations and recent multi-wavelength discoveries, including the Fermi LAT pulsar PSR J1022-5746 and giant molecular clouds in the region. Despite the improved VHE dataset, a clear identification of the object responsible for the VHE emission from HESS J1023-575 is not yet possible, and contribution from the nearby high-energy pulsar and/or the open cluster remains a possibility.

Key words. gamma rays: stars - HII regions

\section{Introduction}

The HESS (High Energy Stereoscopy System) collaboration reported the detection of a bright, extended (standard deviation $\sigma=0.18^{\circ}$ ) VHE $\gamma$-ray source HESS J1023-575

\footnotetext{
* Supported by CAPES Foundation, Ministry of Education of Brazil.
}

(Aharonian et al. 2007a) coincident with the open stellar cluster Westerlund 2. This discovery provides evidence for particle acceleration to extremely high energies in this region. Young stellar clusters are among the sites considered for high-energy $\gamma$-ray production. This relates to the existence of late states of stellar evolution, which are generally considered favorable for 
particle acceleration. Different scenarios have been proposed to account for VHE $\gamma$-ray emission from stellar environments, considering the contribution of hot and massive Wolf-Rayet (WR) and OB stars and their winds in single, binary, or collective processes, pulsars and their synchrotron nebulae, as well as supernova explosions and their expanding remnants (Eichler \& Usov 1993; White \& Chen 1995; Benaglia \& Romero 2003; Reimer et al. 2006; Pittard \& Dougherty 2006; Torres et al. 2004; Bednarek 2005; Manolakou et al. 2007). The giant H II region RCW 49 (NGC 3247) and its ionizing cluster Westerlund 2, hosting over two dozen of massive stars as well as two remarkable WR stars (WR 20a and WR 20b), have been studied extensively over the whole electromagnetic spectrum, and certainly interest was further boosted by the discovery of VHE $\gamma$-ray emission from the vicinity of Westerlund 2.

The VHE $\gamma$-ray source HESS J1023-575 (Aharonian et al. 2007 a) is characterized by a power-law spectrum with a photon index $\Gamma \approx 2.5$ and, at a nominal distance of $8 \mathrm{kpc}$ (obtained from timing studies of the WR binary WR 20a; Rauw et al. $2007)$, would have a $\gamma$-ray luminosity $L_{\gamma}(E>380 \mathrm{GeV})=1.5 \times$ $10^{35} \mathrm{erg} \mathrm{s}^{-1}$. The extent of the observed VHE $\gamma$-ray emission spatially overlaps with the wind-blown bubbles observed in infrared (IR) by Spitzer (Whitney et al. 2004) and in radio continuum by ATCA at 1.38 and $2.38 \mathrm{GHz}$ (Whiteoak \& Uchida 1997). One of these bubbles, with a diameter of $\sim 7.3^{\prime}$ is believed to be connected to the massive binary WR 20a, surrounding the central region of Westerlund 2, while the second has been related to WR $20 \mathrm{~b}$, with a diameter of $\sim 4.1^{\prime}$. The WR binaries WR 20a and WR 20b have been detected in X-rays in the 0.5 to $10 \mathrm{keV}$ band with Chandra (Nazé et al. 2008) with luminosities of $19 \times 10^{32} \mathrm{erg} \mathrm{s}^{-1}$ and $52 \times 10^{32} \mathrm{erg} \mathrm{s}^{-1}$ respectively. The high X-ray luminosity seen from WR 20a, which is likely associated with the open cluster, provides support for the scenario whereby in a colliding wind zone energy dissipated in shocks is converted into thermal energy (Nazé et al. 2008). The observed source extension $\left(0.18^{\circ}\right)$ and nonvariability of the $\gamma$-ray flux of HESS J1023-575 disfavor any scenario where particle acceleration occurs close to the massive stars, and in the colliding wind zone within the massive WR 20a binary system scenario. A prominent feature at radio wavelengths, known as the "blister" (Whiteoak \& Uchida 1997), coincident with HESS J1023-575 (Aharonian et al. 2007a), does not seem to be evident in the diffuse X-ray flux measured by Chandra (Nazé et al. 2008), which appears featureless and predominantly of thermal origin. Upper limits on the non-thermal diffuse emission were recently reported by Fujita et al. (2009) in the $0.7-2 \mathrm{keV}\left(F_{\mathrm{ul}}<2.6 \times 10^{-12} \mathrm{erg} \mathrm{cm}^{-2} \mathrm{~s}^{-1}\right)$ and 0.7 to $10 \mathrm{keV}$ $\left(F_{\mathrm{ul}}<6.1 \times 10^{-12} \mathrm{erg} \mathrm{cm}^{-2} \mathrm{~s}^{-1}\right)$ bands, using Suzaku observations and integrating over the entire camera field $\left(17.8^{\prime} \times 17.8^{\prime}\right)$. According to Fujita et al. (2009), the statistics are not sufficient to distinguish between three thermal components and two thermal components plus a non-thermal one when modeling the background. They claim that their multicomponent fit supports the existence of non-thermal emission. However, the ambiguity in the goodness-of-fit using a multi-thermal component and a multi-thermal component including a non-thermal one does not warrant such a conclusion on the basis of the presented data.

A possible scenario discussed to explain the VHE $\gamma$-ray emission includes the interaction of cosmic-ray protons accelerated in stellar wind shocks or supernova blast waves with dense molecular clouds (Aharonian \& Atoyan 1996). The molecular cloud content in this region observed by the CfA $1.2 \mathrm{~m}$ Millimeter-Wave Telescope and the 4-m Nanten 2 sub-mm telescope shows a complex and rich environment. Dame (2007) observed Westerlund 2 with the CfA $1.2 \mathrm{~m}$ telescope using ${ }^{12} \mathrm{CO}(J=1-0)$ survey data and found a possible association with a giant molecular cloud (GMC) on the far side of the Carina spiral arm, at a distance of $5.0 \mathrm{kpc}$. Observations at a higher resolution $\left(90^{\prime \prime}\right)$ on the $J=2-1$ transition of ${ }^{12} \mathrm{CO}$ performed by the Nanten 2 4-m sub-millimeter telescope (Furukawa et al. 2009; Ohama et al. 2010) confirm the Dame (2007) results but also suggest another possible association. A significant clump of molecular gas, consisting of a foreground component at $0 \mathrm{~km} \mathrm{~s}^{-1}$ is found, with a mass of $(9.1 \pm 4.1) \times 10^{4} M_{\odot}$, and a background component at $16 \mathrm{~km} \mathrm{~s}^{-1}$, with a mass of $(8.1 \pm 3.7) \times 10^{4} M_{\odot}$, both of which are claimed to be connected with RCW 49, at a distance of $5.4_{-1.4}^{+1.1} \mathrm{kpc}$. The analysis of the Nanten survey in the ${ }^{12} \mathrm{CO}(J=1-0)$ (Fukui et al. 2009) also shows two unusual structures in the vicinity of the line-of-sight projection: a straight feature in the East and an arc-like feature in the West in the velocity range $20-30 \mathrm{~km} \mathrm{~s}^{-1}$, corresponding to a distance of $\sim 7 \mathrm{kpc}$ (using the rotation curve of Brand \& Blitz 1993), although the association with the open cluster is unclear.

At high energies (HE; $E>100 \mathrm{MeV}$ ) the Fermi Large Area Telescope (LAT) collaboration has recently reported the detection of two new sources: 1FGL J1023.0-5746, positionally coincident with HESS J1023-575, and a new $\gamma$-ray pulsar 1FGL J1028.4-5810 (PSR J1028-5819) to the east of the HESS source and associated with the EGRET source 3EG J1027-5817 (Abdo et al. 2009a).

The first source, reported in the bright source list, has been very recently identified with a new $\gamma$-ray pulsar, PSR J10225746 (Dormody et al. 2009; Abdo et al. 2010; Saz Parkinson et al. 2010). The pulsar periodicity found in the blind search performed by the Fermi LAT collaboration is $111.47 \mathrm{~ms}$. The young pulsar (with a characteristic age of $\tau \sim 4.6 \mathrm{kyr}$ ) has a spin-down luminosity of $1.1 \times 10^{37} \mathrm{erg} \mathrm{s}^{-1}$. Radio pulsations have not been found but the $130 \mathrm{ks}$ Chandra image shows a faint source (CXOU J102302.8-574606.9, Tsujimoto et al. 2007) identified as a possible counterpart. The second source, PSR J1028-5819 (Abdo et al. 2009a; Manchester et al. 2005) is a $91.4 \mathrm{~ms} \gamma$-ray and radio pulsar with a total spin-down power of $\dot{E}=8.3 \times 10^{35} \mathrm{erg} \mathrm{s}^{-1}$ and a phase-average luminosity from 0.1 to $30 \mathrm{GeV}$ of $(120 \pm 73) \times 10^{33} \mathrm{erg} \mathrm{s}^{-1}$ at a dispersion measure-derived distance of $2.3 \pm 0.7 \mathrm{kpc}$. Its characteristic age is $8.9 \times 10^{4} \mathrm{yr}$. The HE photon spectrum shows a hard cutoff at $1.9 \pm 0.5 \mathrm{GeV}$, while no contribution of steady emission is reported.

The unresolved origin of the VHE $\gamma$-ray emission from the direction of Westerlund 2 motivated further observations, aiming to discriminate among the alternative scenarios for the VHE emission. The results of these deep observations, alongside the discovery of a new, hard, VHE $\gamma$-ray emission coincident with the Fermi LAT source PSR J1028-5819, are reported here. The nature of the VHE sources is then discussed in light of the new multi-wavelength observations in the Westerlund 2 field.

\section{Analysis and results}

The High Energy Stereoscopic System (HESS) is an array of four VHE $\gamma$-ray imaging atmospheric Cherenkov telescopes (IACTs) located in the Khomas Highland of Namibia $\left(23^{\circ} 16^{\prime} 18^{\prime \prime} \mathrm{S} 16^{\circ} 30^{\prime} 00^{\prime \prime} \mathrm{E}\right)$. Each of these telescopes is equipped with a tessellated spherical mirror of $107 \mathrm{~m}^{2}$ area and a camera comprised of 960 photomultiplier tubes, covering a large fieldof-view (FoV) of $5^{\circ}$ diameter. Its large FoV and good off-axis sensitivity make HESS ideally suited for studies of extended sources and regions of the sky in which more than one $\gamma$-ray 
source could be present (see e.g. Aharonian et al. 2008). The system works in a coincidence mode (e.g. Funk et al. 2004), requiring at least two of the four telescopes to trigger the detection of an extended air shower (EAS). This stereoscopic approach results in a high angular resolution of $\sim 5^{\prime}$ per event, good energy resolution ( $6 \%$ on average) and an effective background rejection (Aharonian et al. 2006b). These characteristics allow HESS to reach a sensitivity $\sim 2.0 \times 10^{-13} \mathrm{ph} \mathrm{cm}^{-2} \mathrm{~s}^{-1}$ (equivalent to $1 \%$ of the Crab Nebula flux above $1 \mathrm{TeV}$ ), or less if advanced techniques are used for image analysis (Acero et al. 2009), for a point-like source detected at a significance of $5 \sigma$ in $25 \mathrm{~h}$ of observation at zenith.

Previous observations of the region containing PSR J10285819 and Westerlund 2 (Aharonian et al. 2007a) were performed between March and July 2006, amounting to a total of $12.9 \mathrm{~h}$ of live time. The analysis of the data led to the discovery of an extended source $\left(\sigma=0.18^{\circ} \pm 0.02^{\circ}\right)$ located at $\alpha=10^{\mathrm{h}} 23^{\mathrm{m}} 18^{\mathrm{s}}$ and $\delta=-57^{\circ} 45^{\prime} 50^{\prime \prime}$ (J2000). The morphological and spectral analyses of the source did not allow a firm identification of the origin of the VHE emission. Energy-dependent morphology studies have proven to be a powerful tool to distinguish between alternative source models and respective VHE $\gamma$-ray emission signatures (Aharonian et al. 2006a). In order to discriminate among the different $\gamma$-ray emission scenarios proposed and to investigate energy-dependent morphology, follow-up observations were pursued. The region was observed for an additional $33 \mathrm{~h}$ in January and March 2007, April and May 2008, and May to June 2009. The complete data set is presented here and has a live time of $45.9 \mathrm{~h}$ after quality cuts to remove data taken during bad weather conditions or hardware irregularities (Aharonian et al. 2006b). The data were taken in wobble-mode configuration, where the telescopes are pointed offset (from $0.5^{\circ}$ to $2.5^{\circ}$ in this data set) from the nominal source location to allow simultaneous background estimation. The zenith angles range from $30^{\circ}$ to $55^{\circ}$, with a mean value of $38^{\circ}$, which results in a mean energy threshold of $\sim 0.8 \mathrm{TeV}$ when using hard cuts on the EAS image size of 200 photoelectrons (p.e.). The mean energy threshold of the new data set is slightly higher than the one in the previous analysis due to lower reflectivity of the IACT mirrors compared to the 2006 observations and due to a stricter cut on the image size than the one used for the 2006 data set (standard cuts at 80 p.e.). The optical response of the system was estimated from the Cherenkov light of single muons hitting the telescopes as explained in Aharonian et al. (2006b).

The data have been analyzed using the HESS standard event reconstruction scheme (Aharonian et al. 2006b) using the Hillas second moment method (Hillas 1985). A second independent analysis chain was used to cross-check the results, including independent calibration of pixel amplitudes and identification of problematic or dead pixels in the IACTs cameras, leading to compatible results. To achieve a maximum signal-to-noise ratio and to improve angular resolution, hard cuts were used to reduce the data, and only observations runs (of 28 min duration) with four active IACTs were used to study the morphology and spectrum of the source.

The large energy range covered by HESS allows the investigation of the morphology and spectral features in the region of interest in different energy bands. For this analysis, two energy bands were selected a priori such that each band has a similar signal-to-noise ratio, for the range of zenith angle of the current observations, assuming the source has a photon index of 2.2 (a typical value for Galactic VHE $\gamma$-ray sources). Images of the VHE $\gamma$-ray excess (see next section), corrected by the

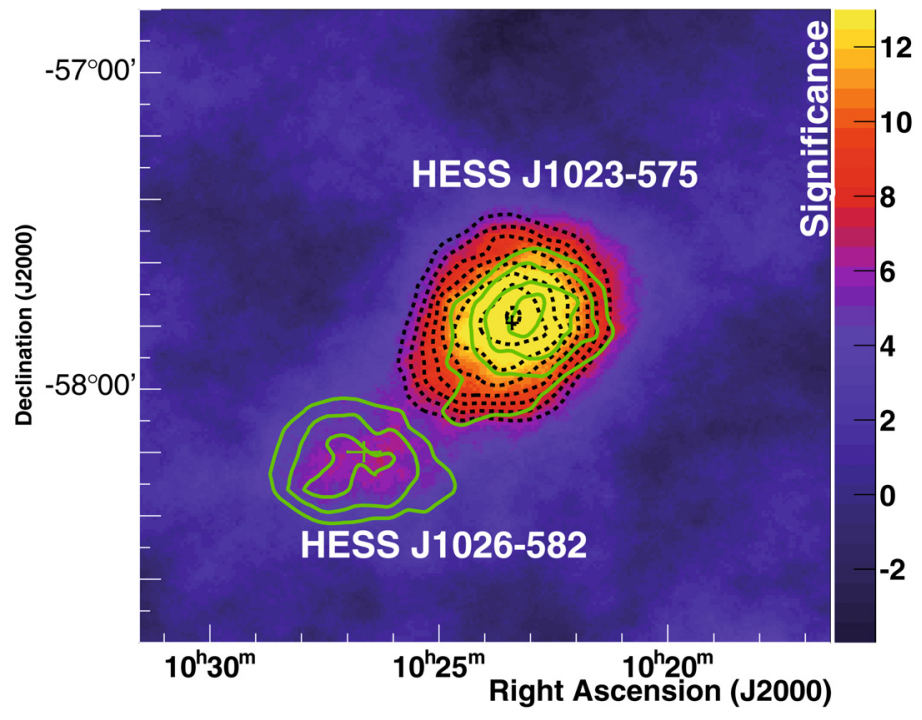

Fig. 1. Image of the Westerlund 2 field showing the significance of the VHE $\gamma$-ray emission using an oversampling radius of $0.22^{\circ}$. The contours in black (dashed) and green (solid) show the significance levels for the maps at low and high energy range, respectively, using the same oversampling radius, starting at $5 \sigma$ in steps of $1 \sigma$. The maximum of the color scale has been chosen such that the typical detection threshold $\sim 5 \sigma$ appears on the transition from blue to red.

corresponding radial acceptance in each energy band, were produced for two bands $(0.7<E<2.5 \mathrm{TeV}$ and $E \geq 2.5 \mathrm{TeV})$.

\subsection{Energy-dependent morphology}

Figure 1 shows the significance map of the region using an oversampling radius of $0.22^{\circ}$ (optimized for extended sources, Aharonian et al. 2006c). The background in each bin of the image was estimated using a ring with a mean radius of $1^{\circ}$ (as defined in Aharonian et al. 2006b) around the test position. The previously reported source HESS J1023-575 is detected with a peak significance of $16 \sigma$ corresponding to 545 excess events above background. A second excess appears to the south-east towards the direction of PSR J1028-5819 (dubbed HESS J1026582 ), showing a peak significance of $7 \sigma$ pre-trials (169 excess events). Source locations are extracted from the uncorrelated excess map fitted to a two-dimensional Gaussian function folded with the HESS point spread function (PSF; $\sim 0.08^{\circ}$ above 200 p.e.). The best fit position lies at $\alpha=10^{\mathrm{h}} 23^{\mathrm{m}} 24^{\mathrm{s}} \pm$ $7.2_{\text {stat }}^{\text {s }}$ and $\delta=-57^{\circ} 47^{\prime} 24^{\prime \prime} \pm 1^{\prime} 12^{\prime \prime}{ }_{\text {stat }}$ (J2000) for HESS J1023575 , compatible with the previously reported position. For the second source, HESS J1026-582, the Gaussian peaks at $\alpha=$ $10^{\mathrm{h}} 26^{\mathrm{m}} 38.4^{\mathrm{s}} \pm 21.6_{\text {stat }}^{\mathrm{s}}$ and $\delta=-58^{\circ} 12^{\prime} 00^{\prime \prime} \pm 1^{\prime} 48^{\prime \prime}{ }_{\text {stat }}(\mathrm{J} 2000)$, $0.6^{\circ}$ apart from the other source. The systematic error is estimated to be $20^{\prime \prime}$ per axis (Gillessen 2004). The two derived positions and statistical errors are marked with black and green crosses in Fig. 1.

To further investigate the nature of the two detected excesses, the image has been analyzed in the two energy bands described above. For the lower energy band, between 0.7 and $2.5 \mathrm{TeV}$, the image (Fig. 2) shows a distinct excess emission at the location of HESS J1023-575, extended beyond the nominal size of the HESS instrument PSF, with an intrinsic extension of $\sigma=0.18^{\circ} \pm 0.02^{\circ}$. The second excess is strongly reduced in this energy band. However, this second emission region, 

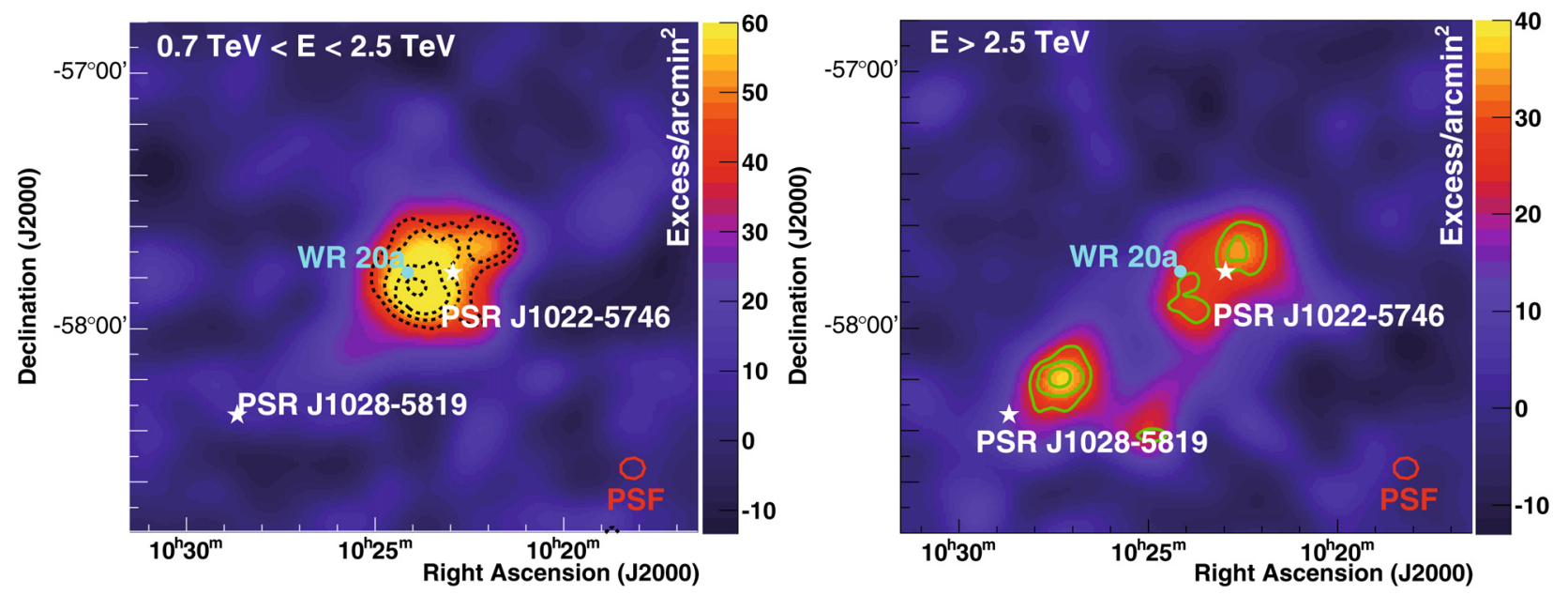

Fig. 2. Gaussian-smoothed $\left(\sigma=0.08^{\circ}\right)$ excess images for the Westerlund 2/RCW 49 and PSR J1028-5819 region. On the left, the low energy map $(0.7 \mathrm{TeV}<E<2.5 \mathrm{TeV})$ is shown, while the high energy map $(E \geq 2.5 \mathrm{TeV})$ is displayed on the right. The HESS significance contours (dashed black lines for the low energy map and solid green ones for the high energy map) are calculated using an oversampling radius of $0.1^{\circ}$ and are shown above $4 \sigma$ in steps of $1 \sigma$. The position of WR 20a and the two Fermi LAT pulsars are marked in cyan and white (for more details see text).

HESS J1026-582, is clearly visible on the high energy image $(E \geq 2.5 \mathrm{TeV})$, indicative of a very hard photon index and also evidence for extension at the scale $\sigma=0.14^{\circ} \pm 0.03^{\circ}$. The two Gaussian-smoothed $\left(\sigma=0.08^{\circ}\right)$ excess maps are shown in Fig. 2 with significance contours above $4 \sigma$ in black dashed lines for the low energy map and solid green lines for the high energy one, obtained by using an integration radius of $0.1^{\circ}$. The significance contours for a larger integration radius $\left(0.22^{\circ}\right)$ for the lower (in black) and the higher (in green) energy bands and are also shown in Fig. 1 together with the total significance map above 200 p.e.

To further investigate the interplay between the two neighboring sources as of HESS J1023-575 and HESS J1026-582, a rectangular region (slice) is defined in the uncorrelated excess maps along the line connecting the best-fitted positions of the two spots with a width of twice the HESS PSF (see Fig. 3 inset). The profile resulting from a projection of the slice on the long axis is shown in Fig. 3 for the low (in red) and high (in blue) energy images. The radial profile for the low energy map is well fitted by a single Gaussian function $\left(\chi^{2} / v=5.57 / 6\right.$, corresponding to a probability of $P=0.47$ ) centered at a position compatible with the centroid of HESS J1023-575 and the position of Westerlund 2 and PSR J1022-5746 (cyan and white markers respectively in the inset figure). Their positions with respect to the center of the slice are marked with dashed lines in the profile figure. However, the profile corresponding to the high energy events shows a second peak towards the direction of PSR J1028-5819 (dashed line) and a fit to a double Gaussian function is clearly favored $\left(\chi^{2} / v=0.45 / 4, P=0.97\right)$ against a single Gaussian one $\left(\chi^{2} / v=10.88 / 6, P=0.09\right)$.

Based on the limited statistics at $E \geq 2.5 \mathrm{TeV}$, the peak of the VHE emission related to HESS J1023-575 appears to shift towards the location of the LAT pulsar PSR J1022-5746 at higher energies although the statistic is too scarce to resolve further energy-dependent morphologies. The angular separation of the two best-fitted positions for the low and high energy images is $\sim 0.08^{\circ}$, of the order of the HESS PSF.

\subsection{Spectral analysis}

Spectral information has been obtained in the following way: two circular regions were defined around the respective centroids of HESS J1023-575 and HESS J1026-582, with radius $0.33^{\circ}$.
The background was evaluated by using the reflected background method, in which symmetric regions, not contaminated by the sources, are used to extract the background (Aharonian et al. 2006b). The energy spectra are derived by means of a forward-folding maximum likelihood fit (Piron et al. 2001).

The two energy spectra are shown in Fig. 4. The spectrum of the region containing HESS J1023-575 is well fit by a power law function $\mathrm{d} N / \mathrm{d} E=N_{0}(E / 1 \mathrm{TeV})^{-\Gamma}$, with a photon index of $\Gamma=2.58 \pm 0.19$ stat \pm 0.2 sys and a normalization constant of $N_{0}=\left(3.25 \pm 0.50_{\text {stat }}\right) \times 10^{-12} \mathrm{TeV}^{-1} \mathrm{~cm}^{-2} \mathrm{~s}^{-1}$ (red markers and line), confirming the results from the previous analysis (dashed line) (Aharonian et al. 2007a). The previously measured flux level was slightly higher but this difference is explained by the larger integration radius $\left(0.39^{\circ}\right)$ used previously. At high energies the previous flux of HESS J1023-575 was contaminated with some fraction of the events related to the new source HESS J1026-582. The energy spectrum of HESS J1026-582 is also well represented by a power law function (blue markers and line), showing a hard photon index of $\Gamma=1.94 \pm 0.20$ stat \pm 0.2 sys as expected from the energy maps, with a normalization constant of $N_{0}=\left(0.99 \pm 0.34_{\text {stat }}\right) \times 10^{-12} \mathrm{TeV}^{-1} \mathrm{~cm}^{-2} \mathrm{~s}^{-1}$. The systematic error on the normalization constant $N_{0}$ is estimated from simulated data to be $20 \%$ (Aharonian et al. 2006b).

The two measured photon indices in the energy range between 1 and $10 \mathrm{TeV}$ differ by $\Delta \Gamma=0.7$, which taking into account their statistical errors, lead to an incompatibility of $2.5 \sigma$ for the two VHE emission regions. The different spectral indices as well as the clear separation of the two sources in the images favor the interpretation of the two emission regions as two independent sources rather than having a common origin.

\section{Discussion}

Follow up observation on the previously discovered source HESS J1023-575 lead to confirmation of the features of this source as well as the discovery of a new VHE $\gamma$-ray source, HESS J1026-582. The two TeV emission regions appear spatially distinct and show different spectral characteristics, favoring an interpretation of being two independent sources. Accordingly, different scenarios regarding the nature of both sources in the context of being related to energetic pulsars or massive stars and their winds are discussed in the following. 


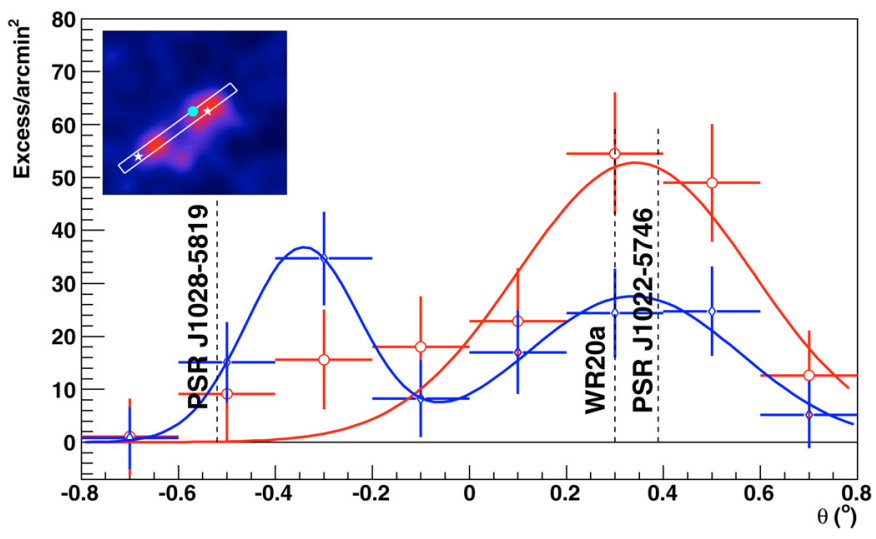

Fig. 3. Profile of the $\mathrm{TeV}$ emission along the slice defined to contain the maximum of the two $\gamma$-ray excesses, as illustrated in the inset. The width of the slice is defined to be twice the HESS angular resolution. The profile on the low energy image is shown in red while the one for the high energy image is shown in blue. The relative positions of WR 20a and the two Fermi LAT pulsars with respect to the center of the slice are indicated by dashed lines.

\subsection{Emission scenarios related to pulsars and their winds}

The spatial coincidence with recently discovered energetic $\gamma$-ray pulsars motivates the investigation of scenarios where $\gamma$-ray pulsars are powering a relativistic wind nebula. In this scenario particles are accelerated to very high energies along their propagation into the pulsar surroundings or at the shocks produced in collisions of the winds with the surrounding medium. As a result of the interactions of relativistic leptons with the magnetic field and low energy radiation (of synchrotron origin, thermal, or microwave background), non-thermal radiation is produced from the lowest possible energies up to $\sim 100 \mathrm{TeV}$. On the other hand, for magnetic fields of a few $\mu \mathrm{G}$, young electrons create a small synchrotron nebula around the pulsar which should be visible in $\mathrm{X}$-rays, in contrast to a much larger $\mathrm{TeV}$ nebula, generated by the inverse Compton (IC) process (for a recent review see Gaensler $\&$ Slane 2006). Typically only pulsars with high spin-down energy $\left(\sim 10^{33} \mathrm{erg} \mathrm{s}^{-1}\right.$, Kargaltsev \& Pavlov 2010) produce prominent pulsar wind nebulae (PWNe). Both pulsars reported from Fermi LAT observations at energies below the HESS detection threshold show high spin-down luminosity and features similar to other pulsars previously associated with VHE $\gamma$-ray sources such as HESS J1825-137 (Aharonian et al. 2006a).

\subsubsection{HESS J1023-575}

The LAT-discovered $\gamma$-ray pulsar PSR J1022-5746 is the youngest and most energetic among the $\gamma$-ray only pulsars known to date, being only one order of magnitude weaker than the Crab pulsar. An X-ray source, CXOU J102302.8-574606.9 (Tsujimoto et al. 2007), found in a $130 \mathrm{ks}$ Chandra observation has been suggested as a potential counterpart, $8^{\prime}$ apart from the Westerlund 2 core, and its relation to the stellar cluster thus remains unknown. A distance to CXOU J102302.8-574606.9 of $>10 \mathrm{kpc}$ was proposed based on the column density from Chandra observations (Dormody et al. 2009). The fitted column density $N_{\mathrm{H}} \sim 1.3 \times 10^{22} \mathrm{~cm}^{-3}$ indicates a large distance $d>8 \mathrm{kpc}$, although given the uncertainties in the measurement and the large absorption expected in the line of sight towards Westerlund 2, a possible association with the open cluster cannot be disregarded. On the other hand, no extended synchrotron PWN has been found yet albeit Suzaku observation time was

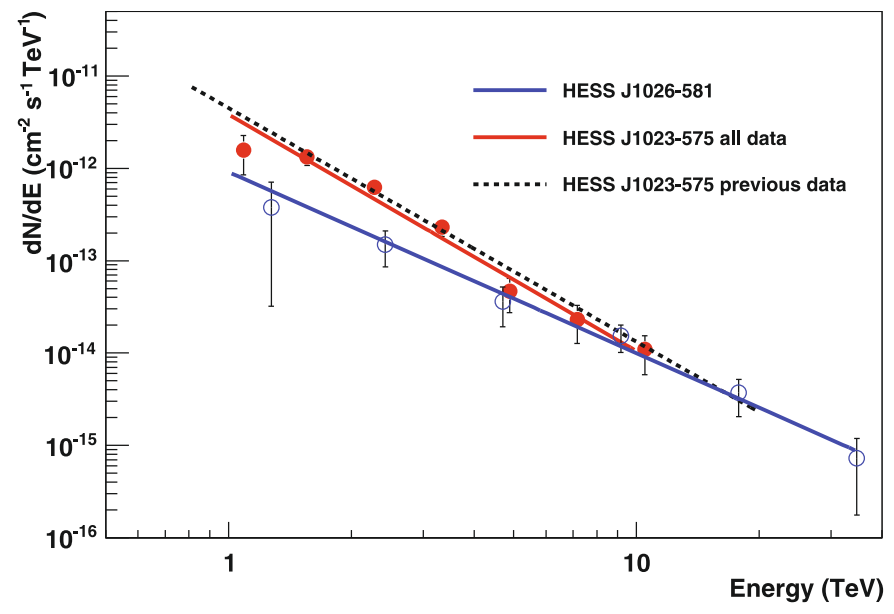

Fig. 4. Differential energy spectra of HESS J1023-575 (red filled circles) and dashed black line for the previous detection and HESS J1026582 (Aharonian et al. 2007a) (blue open circles).

dedicated to directly investigate this hypothesis (Fujita et al. 2009). As noted by Fujita et al. (2009) the ratio of TeV to Xray luminosity is larger than one, assuming that the size of the $\mathrm{X}$-ray emission they considered to obtain upper limits on the diffuse non-thermal emission in the 0.7 to $10 \mathrm{keV}$ range corresponds to the same emitting region at VHE. That would suggest that the population of $\mathrm{TeV}$ emitting particle has already cooled and does not shine significantly in X-ray synchrotrons as is the case of most middle aged $\mathrm{TeV}$ nebulae (de Jager \& Djannati-Ataï 2008).

With its high spin-down luminosity, the Fermi LAT pulsar is sufficiently strong to power the observed VHE $\gamma$-ray emission. Assuming the pulsar is at a distance of $8 \mathrm{kpc}$, the luminosity of the HESS source in the 1 to $10 \mathrm{TeV}$ range is $4.8 \times 10^{34} \mathrm{erg} \mathrm{s}^{-1}$. The association with the pulsar would imply conversion from rotational energy into non-thermal emission with efficiency $0.4 \%$, comparable to those inferred for other VHE $\gamma$-ray sources associated with PWNe, i.e., HESS J1912+1011 (Aharonian et al. 2008) or HESS J1718-385 (Aharonian et al. 2007b). However, the pulsar distance and age limit the maximum extension of the nebula (see i.e. Kennel \& Coroniti 1984) and render it difficult to explain the total extension of the $\mathrm{TeV}$ emission as due to inverse Compton scattering of the PWN on the interstellar radiation field (Gaensler \& Slane 2006; de Jager \& Djannati-Ataï 2008). The projected size of HESS J1023-575 corresponds to $\sim 28 \mathrm{pc}(d / 8.0 \mathrm{kpc})$ (i.e. 6 or $31 \mathrm{pc}$ assuming distances of 2 and $10 \mathrm{kpc}$, respectively). An estimation of the maximum size of the nebula is given by Kennel \& Coroniti (1984) (KC model):

$$
\begin{aligned}
R \sim 2 & \times 10^{20}\left(\frac{L_{\mathrm{SD}}}{10^{37} \mathrm{erg} / \mathrm{s}}\right)^{1 / 3}\left(\frac{t}{10^{5} \mathrm{yr}}\right)^{1 / 3} \\
& \times\left(\frac{p_{\mathrm{ism}}}{10^{-12} \mathrm{erg} / \mathrm{cm}^{3}}\right)^{-1 / 3} \mathrm{~cm} \sim 19 \mathrm{pc}
\end{aligned}
$$

obtained by balancing the total luminosity at present provided by the pulsar against the interstellar medium pressure. The obtained value is 1.5 times smaller than the estimated size of the HESS source for a pulsar as far as $8 \mathrm{kpc}(28 \mathrm{pc})$, although this value has a strong dependency on the assumed input parameters. Under these assumptions (using the pulsar parameters and a typical pressure for the ISM of $p_{\text {ism }} \sim 2 \times 10^{-12} \mathrm{eV} \mathrm{cm}^{-3}$ ), the KC PWN model for a pulsar located at the nominal distance of Westerlund 2 does not describe well the VHE emission 
Table 1. Properties of high energy pulsars associated to the TeV sources and inferred TeV luminosity for the nominal pulsar distance.

\begin{tabular}{ccccccc}
\hline \hline HESS source & Pulsar name & $\dot{E}\left[\mathrm{erg} \mathrm{s}^{-1}\right]$ & $d_{\text {nom }}[\mathrm{kpc}]$ & $\tau[\mathrm{kyrs}]$ & $B\left[10^{12} \mathrm{G}\right]$ & $L_{\gamma}\left(d / d_{\text {nom }}\right)^{2}(1-10 \mathrm{TeV})\left[10^{33} \mathrm{erg} \mathrm{s}^{-1}\right]$ \\
\hline HESS J1023-575 & PSR J1022-5746 & $1.1 \times 10^{37}$ & 8 & 4.6 & 6.6 & 48 \\
HESS J1026-582 & PSR J1028-5819 & $8.3 \times 10^{35}$ & 2.3 & 89 & 1.2 & 2.5 \\
\hline
\end{tabular}

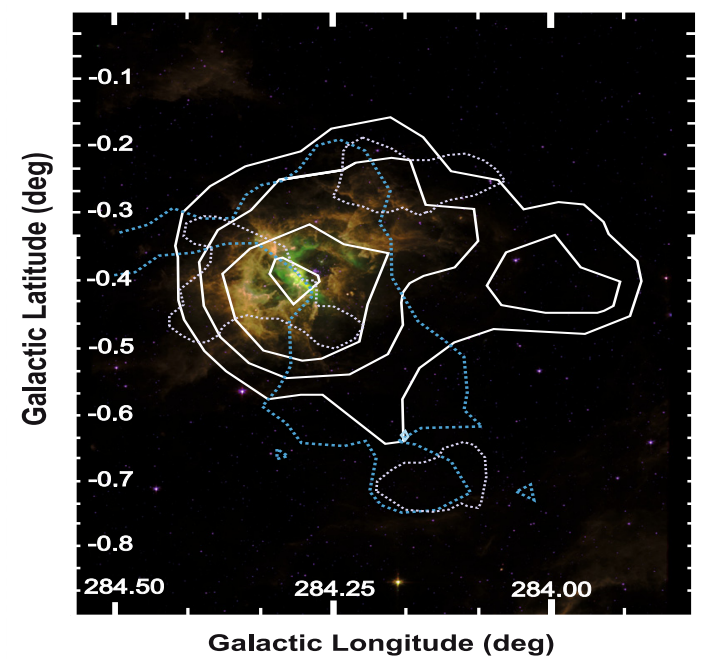

Fig. 5. Spitzer/IRAC GLIMPSE three-color image (in red the $5.8 \mu \mathrm{m}$ image, $8 \mu \mathrm{m}$ in green and $3.6 \mu \mathrm{m}$ in blue) of RCW 49 overlaid with ${ }^{12} \mathrm{CO}(J=2-1)$ contours at velocity ranges of 10.8 to $20.9 \mathrm{~km} \mathrm{~s}^{-1}$ and 1.2 to $8.7 \mathrm{~km} \mathrm{~s}^{-1}$ observed with the Nanten 2 telescope (in blue and purple respectively). The white contours show the HESS significance derived for a correlation radius of $0.1^{\circ}$, hard cuts and a maximum energy of $2.5 \mathrm{TeV}$. The lowest contour corresponds to $4 \sigma$.

and more sophisticated scenario are needed, including possible anisotropy of the pulsar wind or evolution of the spin-down luminosity which will allow a higher intrinsic spin-down energy. Also, the presence of stellar winds from $\mathrm{O}$ type stars are known to create cavities, allowing a larger PWN size compared to expansion into more typical ISM pressures. A PWN according to the model of $\mathrm{KC}$ would be consistent with a distance estimate of $\sim 2 \mathrm{kpc}$. Recently, a revised distance was estimated by Saz Parkinson et al. (2010) suggesting PSR J1022-5746 at a distance of $2.4 \mathrm{kpc}$.

\subsubsection{HESS J1026-582}

The association of the VHE $\gamma$-ray source HESS J1026-582 with the Fermi LAT pulsar PSR J1028-5819 $\left(\alpha=10^{\mathrm{h}} 28^{\mathrm{m}} 39.8^{\mathrm{s}}\right.$ and $\delta=-58^{\circ} 17^{\prime} 13^{\prime \prime}$, J2000, with a $95 \%$ confidence level radius of $0.079^{\circ}$ ) seems likely and can be naturally described by interaction of the pulsar wind with the surrounding medium. Moreover, an alternative counterpart has not been proposed so far. The spin-down energy of the pulsar is high enough to power the VHE $\gamma$-ray emission, which, assuming a distance of $2.3 \mathrm{kpc}$, results in a total luminosity in the 1 to $10 \mathrm{TeV}$ energy range of $2.5 \times 10^{33} \mathrm{erg} \mathrm{s}^{-1}$. The implied conversion efficiency from spindown power into particle luminosity would imply a similar value to the one considered above, about $0.3 \%$. No PWN association at X-ray energies has been found so far, although observations with both XMM-Newton and Suzaku have been carried out triggered by the Fermi LAT detection. The corresponding projected extension of HESS J1026-582 is 5.6 pc for an assumed distance of $2.3 \mathrm{kpc}$. Using Eq. (1), the estimated maximum size is $\sim 8 \mathrm{pc}$, which is compatible with the observed extension at VHE energies. The large offset of the pulsar with respect to the center of gravity of the VHE source $\left(0.28^{\circ}\right)$ and asymmetric morphology is similar to other relic TeV PWN such as HESS J1825-137 and can be explained either by the proper velocity of the pulsar or by an asymmetric reverse shock resulting from a supernova that exploded initially in an inhomogeneous medium (Blondin et al. 2001). If the pulsar was born at the HESS source position, a pulsar transverse velocity of $\sim 100 \mathrm{~km} \mathrm{~s}^{-1}$ can be estimated using the dispersion-measured based distance of $2.3 \mathrm{kpc}$, and a characteristic age of $8.9 \times 10^{4} \mathrm{yr}$. This is in the observed range of known pulsar velocities.

A summary of the observed parameters of the high energy pulsars and inferred luminosity above $1 \mathrm{TeV}$ for the nominal distances $\left(d_{\text {nom }}\right)$ of the associated TeV sources is given in Table 1.

\subsection{Emission scenarios related to massive stars and their winds}

The possibility of an association of HESS J1023-575 with the open cluster Westerlund 2 was discussed in the HESS J1023-575 discovery paper (Aharonian et al. 2007a). Different mechanisms involving cosmic-rays accelerated in expanding stellar winds or supernova blast waves interacting with the boundaries of the interstellar radiation field or the boundaries of the radio blister (Voelk 1983) are among the possible scenarios to explain the VHE $\gamma$-ray emission. The total mechanical luminosity $\left(W_{\text {total }}\right)$ available from stellar winds in Westerlund 2 is estimated to be $3.6 \times 10^{51}$ erg (Rauw et al. 2007). Observations of the molecular cloud content in the region by Dame (2007) shows a complex and rich environment and triggered high resolution $\left(1.5^{\prime}\right)$ observations with the Nanten $24-\mathrm{m}$ sub-mm telescope. The latter observations resolved massive molecular clouds at $\sim 16 \mathrm{~km} \mathrm{~s}^{-1}$ and $\sim 4 \mathrm{~km} \mathrm{~s}^{-1}(6.5$ and $5.2 \mathrm{kpc})$, that were previously reported by Dame (2007), as well as a second component at $\sim-4 \mathrm{~km} \mathrm{~s}^{-1}$ which appears to be coincident with a region of ongoing star formation. To illustrate the extent of the star formation region, a three-color Spitzer/IRAC GLIMPSE image is shown in Fig. 5, in red the $5.8 \mu \mathrm{m}$ image, $8 \mu \mathrm{m}$ in green and $3.6 \mu \mathrm{m}$ in blue. The Nanten 2 radio signal reported in the velocity ranges of 10.8 to $20.9 \mathrm{~km} \mathrm{~s}^{-1}$ and 1.2 to $8.7 \mathrm{~km} \mathrm{~s}^{-1}$ is overlaid in blue and purple dashed lines respectively, showing a strong spatial correlation with the cluster. The HESS low energy significance contours are shown in white. These GMCs provide a sufficiently dense target $\left(\sim 8-9 \times 10^{4} M_{\odot}\right.$ which translates to a density of $n \sim 54-60 \mathrm{~cm}^{-3}$ for a size corresponding to the HESS J1023-575 extension at $8 \mathrm{kpc}$ ) for high-energy particle interactions, allowing the production of $\gamma$-rays from $\pi$-decay via inelastic pp-collisions. In both cases, considering the GMC mass and distance, (and using $W_{\mathrm{pp}}=L_{\gamma} \tau_{\mathrm{pp}}(n)$, with $\tau_{\mathrm{pp}}$ being the cooling time for $\mathrm{p}-\mathrm{p}$ interactions) less than $0.05 \%$ of the total mechanical luminosity estimated for the stellar winds in Westerlund 2 would be sufficient to explain the observed VHE emission.

A power-law extrapolation of the HESS spectrum to lower energies (to $30 \mathrm{GeV}$ ) yields a flux of $2.8 \times 10^{-8} \mathrm{TeV}^{-1} \mathrm{~cm}^{-2} \mathrm{~s}^{-1}$ 
(or 1.7-6.2 $\times 10^{-8} \mathrm{TeV}^{-1} \mathrm{~cm}^{-2} \mathrm{~s}^{-1}$ considering statistical errors in the normalization and spectral index). This flux extrapolation is well above the differential flux measured by Fermi LAT at approximately the same energy $\left(\sim 3.3 \times 10^{-9} \mathrm{TeV}^{-1} \mathrm{~cm}^{-2} \mathrm{~s}^{-1}\right.$, Abdo et al. 2010). Thus, the combination of the HESS and Fermi LAT results implies a spectral hardening towards lower energies, which can be described with a turnover of the HESS spectrum at $\sim 100 \mathrm{GeV}$. If hadronic emission processes are responsible for the emission, the lower energy cutoff could result from cosmic-rays of energy $<1 \mathrm{TeV}$ being prevented from reaching the GMC, perhaps via slow diffusion as demonstrated by Aharonian \& Atoyan (1996), where only high energy hadrons reach the surrounding molecular clouds. In this scenario, considering an age for Westerlund 2 of 1 to 2 million years, low diffusion coefficients in the order of $10^{26} \mathrm{~cm}^{2} \mathrm{~s}^{-1}$ are necessary to prevent low energy particles from reaching the $\gamma$-ray production site.

Emission scenarios where the bulk of the VHE $\gamma$-rays is produced close to the massive WR stars WR 20a and/or WR 20b were already disfavored given the observed extension and lack of variability in the VHE $\gamma$-ray emission, as discussed in Aharonian et al. (2007a). However, $\gamma$-rays from collective stellar wind interactions, where energetic particles experience multiple shocks, may still contribute to the observed VHE emission.

\section{Conclusions}

The HESS source HESS J1023-575 has been re-observed in order to search for energy-dependent morphology, that could provide clues on the origin of the $\gamma$-ray emission. The analysis of the new data unveils a second VHE emission peak towards the direction of the energetic high energy pulsar PSR J1028-5819, reported recently from Fermi LAT observations at lower energies $(0.1$ to $\sim 30 \mathrm{GeV})$. The centers of gravity of the two VHE $\gamma$-ray emission regions are well separated $\left(0.6^{\circ}\right)$ and the radial profile shows two clear peaks at the higher energies, while at lower energies the VHE emission corresponding to HESS J1026-582 appears very much suppressed. This new source is detected with a significance level of $7 \sigma$ and is characterized by a very hard photon spectral index of 1.9. HESS J1026-582 is preferably interpreted as due to a PWN energized by PSR J1028-5819, with similar characteristics as those previously detected by HESS (such as hard spectrum photon index, offset of the high energy pulsar with respect the center of the $\mathrm{TeV}$ emission, similar conversion efficiency from rotational energy into non-thermal emission, etc.). Further observations with X-ray satellites of this region will have a chance to provide the required additional information to support and settle the suggested scenario of being another GeV pulsar/TeV PWN association.

The previously reported VHE source HESS J1023-575 has been confirmed and the analysis of the new data shows compatible results. Multi-wavelength observations of the regions provided new insights on the potential origin of the $\gamma$-ray emission as discussed in the previous section. A possible association with the recently discovered $\gamma$-ray PSR J1022-5746 (1FGL J1023.0-5746), is supported by spatial coincidence with HESS J1023-575, and can be easily accommodated in terms of energetics if the pulsar is as close as $2-3 \mathrm{kpc}$, while more sophisticated PWN models are necessary if it is located significantly further away. On the other hand, the molecular content of the region provides sufficient target material to explain the emission through hadronic interaction of cosmic-rays accelerated by a range of feasible mechanisms in the open cluster interacting with molecular clouds.
Whether the emission is due to the not-yet-detected PWN associated to the Fermi LAT pulsar PSR J1022-5746 or mechanisms related to acceleration of cosmic-rays in the open cluster, a spectral flattening at lower energies must be present to explain the lower MeV-GeV flux level. In the first case, IC processes would naturally reproduce the spectral shape, while for hadronic emission, a lower cutoff at a several tens of $\mathrm{GeV}$ would reflect a lower cutoff on the cosmic-ray spectrum at $\sim 1 \mathrm{TeV}$. The latter scenario could be explained by assuming a low diffusion coefficient in the region, which would not be surprising in the context of the cavities created by the stellar winds from the young OB stars in the stellar cluster. Despite the already accomplished multi-wavelength coverage, a firm identification is still pending, whereas the high-power pulsar PSR J1022-5746 is the prime counterpart hypothesis to adopt now. Unfortunately the statistics at VHE is too scarce to distinguish any further hint of energy-dependent morphology which would have the potential to settle the association with the pulsar or the open cluster. A clear identification of a PWN associated to the Fermi LAT pulsar PSR J1022-5746 in X-rays would help to elucidate the real nature of HESS J1023-575, but given the stellar density and richness in X-ray sources towards Westerlund 2, this appears to be a serious observational challenge. In particular deeper VHE observations might allow higher energy resolution studies and a firm identification of the origin of the VHE emission.

Acknowledgements. The support of the Namibian authorities and of the University of Namibia in facilitating the construction and operation of HESS is gratefully acknowledged, as is the support by the German Ministry for Education and Research (BMBF), the Max Planck Society, the French Ministry for Research, the CNRS-IN2P3 and the Astroparticle Interdisciplinary Programme of the CNRS, the U.K. Science and Technology Facilities Council (STFC), the IPNP of the Charles University, the Polish Ministry of Science and Higher Education, the South African Department of Science and Technology and National Research Foundation, and by the University of Namibia. We appreciate the excellent work of the technical support staff in Berlin, Durham, Hamburg, Heidelberg, Palaiseau, Paris, Saclay, and in Namibia in the construction and operation of the equipment.

\section{References}

Abdo, A., Ackermann, M., Ajello, M., et al. (Fermi Collaboration) 2010, ApJS, 188,405

Abdo, A., Ackermann, M., Atwood, W. B., et al. (Fermi Collaboration) 2009a, ApJ, 695, 72

Abdo, A., Ackermann, M., Ajello, M., et al. (Fermi Collaboration) 2009b, ApJS, 183,46

Acero, F., Aharonian, F., Akhperjanian, A. G., et al. (HESS Collaboration) 2009, Science, 326, 1080

Aharonian, F., \& Atoyan, A. M. 1996, A\&A, 309, 917

Aharonian, F., Akhperjanian, A. G., Aye, K.-M., et al. (HESS Collaboration) 2005, A\&A, 442, 1

Aharonian, F., Akhperjanian, A. G., Bazer-Bachi, A. R., et al. (HESS Collaboration) 2006a, A\&A, 460, 365

Aharonian, F., Akhperjanian, A. G., Bazer-Bachi, A. R., et al. (HESS Collaboration) 2006b, A\&A, 457, 899

Aharonian, F., Akhperjanian, A. G., Bazer-Bachi, A. R., et al. (HESS Collaboration) 2006c, ApJ, 636, 777

Aharonian, F., Akhperjanian, A. G., Bazer-Bachi, A. R., et al. (HESS Collaboration) 2007a, A\&A, 467, 1075

Aharonian, F., Akhperjanian, A. G., Bazer-Bachi, A. R., et al. (HESS Collaboration) 2007b, A\&A, 472, 489

Aharonian, F., Akhperjanian, A. G., Barres de Almeida, U., et al. (HESS Collaboration) 2008, A\&A, 484, 435

Bednarek, W. 2005, MNRAS, 363, L46

Benaglia, P., \& Romero, G. E. 2003, A\&A, 399, 1121

Blondin, J. M., Chevalier, R. A., \& Frierson, D. M. 2001, ApJ, 563, 806

Brand, J., \& Blitz, L. 1993, A\&A, 275, 67

Dame, T. M. 2007, ApJ, 665, 163

de Jager, O. C., \& Djannati-Ataï, A. 2008, A\&SS Library, 67, 357

Dormody, M. H., et al. (Fermi Collaboration) 2009, Proceed. Fermi Symposium [arXiv: 0912.3949] 
Eichler, D., \& Usov, V. 1993, ApJ, 40, 271

Fujita, Y., Hayashida, K., Takahashi, H., \& Takahara, F. 2009, PASJ, 61, 1229 Fukui, Y., Furukawa, N., Dame, T. M., et al. 2009, Astron. Soc. Japan, 61, 23 Funk, S., Hermann, G., Hinton, J., et al. 2004, Astropaticle Phys., 22, 285 Furukawa, N., Dawson, J. R., Ohama, A., et al. 2009, ApJ, 696, L115 Gaensler, B. M., \& Slane, P. O. 2006, ARA\&A, 44, 17

Gillessen, S. 2004, Ph.D. Thesis, University of Heidelberg

Kargaltsev, O., \& Pavlov, G. G. 2010, in Proc. X-ray Astronomy 2009 (Bologna, Italy: AIP)

Hillas, A. M. 1985, in Proceedings of the 19th International Cosmic Ray Conference, La Jolla, ed. F. C. Jones, et al. (Washington, D.C.: NASA)

Kennel, C. F., \& Coroniti, F. V. 1984, ApJ, 283, 694

Manchester, R. N., Hobbs, G. B., Teoh, A., \& Hobbs, M. 2005, AJ, 129, 1993

Manolakou, K., Horns, D., \& Kirk, J. G. 2007, A\&A, 474, 689

Nazé, Y., Rauw, G., \& Manfroid, J. 2008, A\&A, 483, 171

Ohama, A., Dawson, J. R., Furukawa, N., et al. 2010, ApJ, 636, 777

Piron, F., Djannati-Ataï, A., Punch, M., et al. 2001, A\&A, 374, 895

Pittard, J. M., \& Dougherty, S. M. 2006, MNRAS, 372, 801

Rauw, G., Manfroid, J., Gosset, E., et al. 2007, A\&A, 463, 981

Reimer, A., Pohl, M. K., \& Reimer, O. 2006, ApJ, 644, 1118

Saz Parkinson, P. M., Dormody, M., Ziegler, M., et al. 2010, ApJ, submitted [arXiv: $1006.2134 \mathrm{v} 1]$

Torres, D. F., Domingo-Santamaria, E., \& Romero, G. E. 2004, ApJ, 601, L75

Tsujimoto, M., Feigelson, E. D., Townsley, L. K., et al. 2007, ApJ, 665, 719

Völk, H. J. 1983, Space Sci. Rev., 36, 3

White, R. L., \& Chen, W. 1995, ed. K. van der Hucht, \& P. M. Williams (Kluwer Academic. Publishers), Proc. IAU Symp., 163, 438

Whiteoak, J. B. Z., \& Uchida, K. I. 1997, A\&A, 317, 563

Whitney, B. A., Indebetouw, R., Babler, B. L., et al. 2004, ApJS, 154, 315

1 Max-Planck-Institut für Kernphysik, PO Box 103980, 69029 Heidelberg, Germany e-mail: emma@mpi-hd.mpg.de

2 Yerevan Physics Institute, 2 Alikhanian Brothers St., 375036 Yerevan, Armenia

3 Centre d'Étude Spatiale des Rayonnements, CNRS/UPS, 9 Av. du Colonel Roche, BP 4346, 31029 Toulouse Cedex 4, France

${ }^{4}$ Universität Hamburg, Institut für Experimentalphysik, Luruper Chaussee 149, 22761 Hamburg, Germany

5 Institut für Physik, Humboldt-Universität zu Berlin, Newtonstr. 15, 12489 Berlin, Germany

6 LUTH, Observatoire de Paris, CNRS, Université Paris Diderot, 5 Place Jules Janssen, 92190 Meudon, France

7 CEA Saclay, DSM/IRFU, 91191 Gif-Sur-Yvette Cedex, France

8 University of Durham, Department of Physics, South Road, Durham DH1 3LE, UK

9 Unit for Space Physics, North-West University, Potchefstroom 2520, South Africa

10 Laboratoire Leprince-Ringuet, Ecole Polytechnique, CNRS/IN2P3, 91128 Palaiseau, France

11 Laboratoire d'Annecy-le-Vieux de Physique des Particules, Université de Savoie, CNRS/IN2P3, 74941 Annecy-le-Vieux, France

12 Astroparticule et Cosmologie (APC), CNRS, Université Paris 7 Denis Diderot, 10, rue Alice Domon et Léonie Duquet, 75205 Paris Cedex 13, France $^{2}$
13 Dublin Institute for Advanced Studies, 5 Merrion Square, Dublin 2, Ireland

${ }^{14}$ Landessternwarte, Universität Heidelberg, Königstuhl, 69117 Heidelberg, Germany

15 Laboratoire de Physique Théorique et Astroparticules, Université Montpellier 2, CNRS/IN2P3, CC 70, Place Eugène Bataillon, 34095 Montpellier Cedex 5, France

16 Universität Erlangen-Nürnberg, Physikalisches Institut, Erwin-Rommel-Str. 1, 91058 Erlangen, Germany

17 Laboratoire d'Astrophysique de Grenoble, INSU/CNRS, Université Joseph Fourier, BP 53, 38041 Grenoble Cedex 9, France

18 Institut für Astronomie und Astrophysik, Universität Tübingen, Sand 1, 72076 Tübingen, Germany

19 LPNHE, Université Pierre et Marie Curie Paris 6, Université Denis Diderot Paris 7, CNRS/IN2P3, 4 Place Jussieu, 75252 Paris Cedex 5, France

20 Charles University, Faculty of Mathematics and Physics, Institute of Particle and Nuclear Physics, V Holešovičkách 2, 18000 Prague 8, Czech Republic

21 Institut für Theoretische Physik, Lehrstuhl IV: Weltraum und Astrophysik, Ruhr-Universität Bochum, 44780 Bochum, Germany

22 University of Namibia, Department of Physics, Private Bag 13301, Windhoek, Namibia

${ }^{23}$ Obserwatorium Astronomiczne, Uniwersytet Jagielloński, ul. Orla 171, 30-244 Kraków, Poland

24 Nicolaus Copernicus Astronomical Center, ul. Bartycka 18, 00-716 Warsaw, Poland

25 School of Physics \& Astronomy, University of Leeds, Leeds LS2 9JT, UK

26 School of Chemistry \& Physics, University of Adelaide, Adelaide 5005, Australia

27 Torun Centre for Astronomy, Nicolaus Copernicus University, ul. Gagarina 11, 87-100 Toruń, Poland

${ }^{28}$ Instytut Fizyki Jạdrowej PAN, ul. Radzikowskiego 152, 31-342 Kraków, Poland

29 Astronomical Observatory, The University of Warsaw, Al. Ujazdowskie 4, 00-478 Warsaw, Poland

30 Institut für Astro- und Teilchenphysik, Leopold-FranzensUniversität Innsbruck, 6020 Innsbruck, Austria

31 Oskar Klein Centre, Department of Physics, Stockholm University, Albanova University Center, 10691 Stockholm, Sweden

32 Oskar Klein Centre, Department of Physics, Royal Institute of Technology (KTH), Albanova, 10691 Stockholm, Sweden

33 Department of Physics and Astronomy, The University of Leicester, University Road, Leicester, LE1 7RH, UK

34 European Associated Laboratory for Gamma-Ray Astronomy, jointly supported by CNRS and MPG

35 National Academy of Sciences of the Republic of Armenia, Yerevan

36 Department of Astrophysics, Nagoya University, Furocho, Chikusaku, Nagoya 464-8602, Japan

\footnotetext{
${ }^{2}$ UMR 7164 (CNRS, Université Paris VII, CEA, Observatoire de Paris).
}

Article number, page 8 of 8 